\title{
Guest Editorial: Ubiquitous Multimedia Systems and Applications
}

\author{
Xiaolong Jin • Ahmed Y. Al-Dubai • Shoukat Ali • \\ Stephen Jarvis
}

Published online: 13 February 2015

(C) Springer Science+Business Media New York 2015

The technologies of computing and communications have undergone a series of evolutionary innovation over the past decades and improved the quality of human life significantly. Ubiquitous computing and communications is a revolutionary paradigm that aims to provide pervasive and reliable computing solutions and communication services anytime and anywhere. This emerging technology is built upon the rapid research and development advances in a wide range of key areas including wireless and sensor networks, mobile and distributed computing, embedded systems, agent technologies, autonomic communication and information security. Ubiquitous computing and communications has drawn significant interests from both academia and industry and continues to attract tremendous research efforts due to its promising new business opportunity in information technology and engineering. This special issue is devoted to papers addressing research challenges and advances towards the design, implementation and evaluation of ubiquitous multimedia computing and communications technologies, systems and applications. This special issue contains six papers, which are briefly introduced as follows.

Exposure fusion is an efficient way for producing high-quality images for common low dynamic range (LDR) output devices from multiple differently exposed LDR images of the same scene, which has significant potential to be applied in the ubiquitous multimedia area. In the paper entitled "A Novel Method for Fusion of Differently Exposed Images Based on Spatial Distribution of Intensity for Ubiquitous Multimedia” (10.1007/s11042-013-1660-0),

X. Jin $(\bowtie)$

CAS Key Lab of Network Data Science and Technology, Institute of Computing Technology, Chinese Academy of Sciences, Beijing, China

e-mail: jinxiaolong@ict.ac.cn

A. Y. Al-Dubai

Edinburgh Napier University, Edinburgh, UK

e-mail: a.al-dubai@napier.ac.uk

S. Ali

IBM, Dublin 4, Ireland

e-mail: shoukat.ali@ie.ibm.com

S. Jarvis

University of Warwick, Coventry, UK

e-mail: s.a.jarvis@warwick.ac.uk 
the authors proposed a novel two-step method for fusing two differently exposed images based on the spatial distribution of intensity. Experimental results demonstrate that the proposed method has better performance and is convenient for GPU realization.

A massively multiplayer online game (MMOG) is a networked game that allows a large number of people to play together. An MMOG is usually hosted on many powerful servers. The "Performance Evaluation and Simulation of Peer-To-Peer Protocols for Massively Multiplayer Online Games" (10.1007/s11042-013-1662-y) paper proposes the methods for designing of $\mathrm{P} 2 \mathrm{P}$-based MMOG protocols. It also evaluates and highlights the performance bottlenecks via simulations.

In the paper entitled "Adaptively Imperceptible Video Watermarking Based on the Local Motion Entropy" (10.1007/s11042-013-1678-3), Li, Chen and Ma developed an adaptively imperceptible video watermarking algorithm using the entropy model for local motion characterization. Through experiments, they demonstrated that using entropy to local motion characteristics can significantly improve the imperceptibility of watermarking, effectively resist common attacks to video watermarking and finally achieve higher robustness.

Image quality is one of the representative requirements of multimedia quality of experience (QoE). Recently, the image quality assessment ranging from 2D images to 3D images has attracted much attention. The "DCT-Based Objective Quality Assessment Metric of 2D/3D Image" (10.1007/s11042-013-1698-z) paper presents an efficient objective image quality assessment metric in block-based discrete cosine transform (DCT) coding, which incorporates the properties of human visual system (HVS) to improve its validity and reliability in evaluating the quality of stereoscopic image. Experimental results demonstrate that the proposed metric is promising in terms of the practical efficiency and reliability for real-life multimedia applications.

Motion detection is an important and hot research issue due to its wide applications and importance in computer vision. However, existing motion detection algorithms cannot achieve flexibility and accuracy simultaneously for good detection results. In the "A Scene-Adaptive Motion Detection Model Based on Machine Learning and Data Clustering" (10.1007/s11042013-1741-0) paper, Hu et al. investigated a scene-adaptive motion detection model based on the technologies of machine learning and clustering. Through experiment, they validated the excellent adaptability and precision of the proposed model.

The last paper entitled "Features of Oculo-Motors and Their Chronological Changes in Response to Varying Sizes of Visual Stimuli" (10.1007/s11042-013-1824-y) presents a chronological accommodation process for oculomotor behaviour using various sizes of visual stimuli and examines the level of performance of estimating viewed sizes using oculomotor indices of the accommodation process. This study suggests that the features of oculomotor indices reflect the size of objects being viewed, and the viewing sizes can be predicted using these features.

Finally, as the guest editors of this special issue, we highly appreciate the Editor-in-Chief, Prof. Borko Furht, for hosting this issue in the Multimedia Tools and Applications journal. We thank the editorial staff of Springer, namely the Senior Editor, Ms. Melissa Fearon, the Editor, Ms. Courtney Clark, and the Journals Editorial Office Assistant, Ms. Angie Malanday, for their excellent support and helpful guidance, without which this special issue would not have been possible. We are very grateful to the authors for their contributions, including those whose papers were not included. Last but not the least, we would like to express our heartfelt thanks to the thoughtful work of many reviewers who provided valuable evaluations and recommendations. 